\title{
Can Zinc correction in SARS-CoV-2 patients improve treatment outcomes?
}

Amir Noeparast ${ }^{1 *}$, Gil Verschelden ${ }^{2 *}$

${ }^{1}$ Laboratory of Molecular Oncology and Department of Medical Oncology, Vrije Universiteit Brussel, Belgium

${ }^{2}$ Department of Infectious Diseases, Erasme Hospital, Université Libre de Bruxelles, Brussels, Belgium

* Equal contribution

Corresponding author: amir.noeparast@vub.be

The ongoing severe acute respiratory syndrome coronavirus 2 (SARS-CoV-2) pandemic for which there is no established treatment available yet, has caused more than 68,000 deaths so far (https://www.worldometers.info/coronavirus/).

SARS-CoV-2 belongs to the family of coronaviruses and has a high genetic similarity to another family member SARS-CoV which caused the first major epidemic of $21^{\text {st }}$ century ${ }^{1,2}$.

SARS-CoV-2 has probably been zoonotically transmitted from their likely host bats to humans via an intermediate host, namely Malayan pangolins ${ }^{3,4}$.

Following the SARS-CoV outbreak in 2003, an Italian group put forward a hypothesis about the efficacy of two old drugs: Chloroquine (CQ) and Hydroxychloroquine (HCQ), against SARS-CoV and its future emergents ${ }^{5}$.

This hypothesis was shown to be relevant in-vitro, first by a Belgian- ${ }^{6}$ and then a Canadian study ${ }^{7}$.

Due to the high genetic similarity of SARS-CoV-2 and SARS-CoV, the hypothesis introduced by Savarino et al. and the further supportive in-vitro evidence served a rational ground for three different Chinese groups to test the efficacy of CQ or HCQ against SARS-CoV-2 in-vitro ${ }^{8-10}$. These studies showed promising in-vitro efficacy of CQ and HCQ against SARS-CoV- $2^{8-10}$.

Unfortunately, in the absence of sufficient clinical data on the (in)efficacy of CQ and HCQ in SARS-CoV-2 patients, the compassionate and off-label use of these medications is becoming politicized.

Herein, we underline some critical features of the CQ/HCQ mechanism of action concerning SARS-CoV-2. 
Moreover, we introduce a hypothesis on a probable link between zinc-deficiency/zinc correction and response to CQ/HCQ- and possibly other SARS-CoV-2 treatments.

\section{SARS-CoV-2 outbreak and reincarnation of (Hydroxy)Chloroquine: ephemeral or long-lasting?}

$\mathrm{CQ}$ and $\mathrm{HCQ}$ have experienced an adventurous journey since their synthesis and a life rich in repurposing, from anti-malaria agents to immunomodulatory medications of several autoimmune disorders ${ }^{11}$.

Following the SARS-CoV epidemic in 2003, Savarino et.al ${ }^{5}$ proposed even a new application for $\mathrm{CQ}$ and $\mathrm{HCQ}$ as potential anti-SARS agents yet.

Savarino et al. considered three lines of pre-SARS era evidence to base on their hypothesis.

$\mathrm{HCQ}$ and $\mathrm{CQ}$ are weak bases. They can be accumulated in the lysosomes and Golgi apparatus and thereby alkalizing their $\mathrm{pH}$. Thus, $\mathrm{HCQ}$ and $\mathrm{CQ}$ can interfere with the $\mathrm{pH}$-dependent endosome-mediated viral entry of several viruses ${ }^{5,11}$.

On the other hand, it was known that CQ could interfere with post-translational modification of some enveloped viruses, their assembly, and budding within the endosomes and trans-Golgi vesicle networks ${ }^{5,11}$.

As both of these mechanisms could be applied to SARS-CoV pathogenesis, Savarino et al. hypothesized that CQ/HCQ could have anti- SARS-CoV effects.

Besides these two antiviral capacities of $\mathrm{CQ}$ and $\mathrm{HCQ}$, Savarino et al. keenly devoted their attention to another feature of these two drugs being their ability to relieve the excessive and damaging antiviral (among others) immune response. Indeed, CQ and HCQ can decrease the production of tumor necrosis factor-alpha (TNF- $\alpha$ ) and interleukin-6, and downregulate both TNF receptors 1 and $2^{5,11}$. As such, CQ and HCQ mitigate macrophage response, monocyte activation, leukocyte extravasation and therefore alleviate the damaging immune response in various tissues, including lung $5,12,13$.

The excessive and damaging immune response could also be one of the consequences of SARSCoV infection. Therefore, Savarino et al. proposed that the immunomodulatory effect of CQ and $\mathrm{HCQ}$ could play an essential role in the battle against SARS-CoV and other emerging coronaviruses in the future 5 .

Notably, today we learn that one of the fatal complications of SARS-CoV-2 in a subgroup of patients is indeed the cytokine storm syndrome and the related acute respiratory distress syndrome (ARDS) ${ }^{14,15}$.

With regards to cytokine storm and ARDS management, recently, Zhou et al. have underlined the HCQ capacity to repress CD154 expressing T-cells, among other inhibitory mechanisms ${ }^{14}$. 
Both CQ and HCQ are reported with such an effect, but Zhou et al. recommend HCQ over CQ for SARS-CoV-2 treatment due to a better safety profile ${ }^{14,16,17}$.

Interestingly, Zhou et al. have also highlighted another mechanism by which HCQ/CQ interferes with coronavirus binding to the host cells, a feature that can contribute to their anti-SARS-CoV2 effects $^{14}$.

Despite promising pre-clinical evidence and intellectual efforts on considering the $\mathrm{CQ} / \mathrm{HCQ}$ as a potential therapy against SARS-CoV-2, the clinical evidence is still far from being conclusive ${ }^{18-23}$.

\section{Hypothesis: Zinc correction in SARS-CoV-2 patients improves HCQ/CQ and other treatments outcomes}

Since the discovery of the first reported case with zinc-deficiency in $\operatorname{Iran}^{24}$ by Prasad et al. in 1961, we have learned a lot about Zinc, and we have much more left to learn.

Zinc is the second most abundant common trace mineral in the human body, with vital biological functions from cell growth and development to cell homeostasis and immune response ${ }^{25,26}$.

Up to a fifth of the global population is estimated to suffer from different degrees of Zinc deficiency ${ }^{27}$. In the western world, Zinc deficiency is more prevalent among the geriatric population $^{26}$ and vegans/vegetarians as well as among people with certain underlying conditions $^{27}$.

Notably, the early reports show that the elderly SARS-CoV-2 patients are among those with a higher fatality rate ${ }^{28}$.

Herein, we summarize three lines of evidence that underscore the significance of Zinc and a possible link with the response to any anti-SARS-CoV-2 treatment, including CQ/HCQ therapy (figure 1).

\section{Zinc deficiency hampers antiviral and antibacterial immune response}

Zinc deficiency has been associated with increased risk of infectious diarrhea and pneumonia among children, while Zinc supplements were shown to have a corrective impact on both complications ${ }^{29}$.

Moreover, zinc deficiency, whether mild or severe, can negatively impact different adult human organs, including the immune system ${ }^{25,26,30}$.

Zinc deficiency is associated with increased risk of developing Staphylococcus aureus pneumonia and streptococcus pneumonia tonsillitis infections ${ }^{30}$ among the elderly population. 
This risk can reduce, upon Zinc correction in the geriatric population ${ }^{25}$. Markedly, both of these respiratory system infections are reported among the fatal co-infections of SARS-CoV- $2^{31}$.

Additionally, zinc is associated with antiviral immune response in general, involving various viruses ${ }^{27}$.

Not surprisingly, yet there is no data available on such a correlation concerning SARS-CoV-2.

It would be essential to learn whether, in particular, zinc-deficient SARS-CoV-2 patients are at higher risk of mortality due to a weak or inefficient cellular and/or humoral immunity in response to SARS-CoV-2.

Further, the potential of combinatorial zinc correction and anti- SARS-CoV-2 treatments such as $\mathrm{CQ} / \mathrm{HCQ}$ could be clinically explored.

\section{Zinc deficiency is associated with cytokine storm}

Zinc-deficiency not only hampers our immune system in terms of lack of efficient response when required ${ }^{30}$, but it can also be associated with an irrelevant and damaging response.

Zinc levels among patients suffering from different auto-immune disorders appear to be lower than the healthy individuals ${ }^{32}$.

Importantly, Zinc deficiency is associated with the excessive and tissue-damaging pro-

inflammatory release of cytokines, including increased tumor necrosis factor-alpha (TNF- $\alpha)^{33,34}$ and IL-635.

As previously mentioned, so far, the TNF- $\alpha$ and IL- 6 have been reported among the usual suspects responsible for lung-damaging and fatal "Cytokine Strom" in a group of SARS-CoV-2 patients.

Notably, Zinc correction is associated with the down-regulation of tumor necrosis factor-alpha 30,33,34.

Concerning IL-6, however, the impact of Zinc might be rather qualitative than quantitative. One study reports that zinc deficiency is associated with increased IL- 6 gene expression in mice ${ }^{36}$, while another study on a human experimental model reports that zinc deficiency does not decrease IL- 6 levels ${ }^{37}$. Nevertheless, it is reported that among the geriatric female population, a gene polymorphism that leads to an increased immune response-mediated release of Zinc is associated with decreased IL- 6 levels ${ }^{38}$. On the other hand, zinc supplementation can even lead to the up-modulation of IL-639.

However, it seems to be a consensus that zinc correction inversely affects the IL-6-mediated response, which is, by nature, a pro-inflammatory response $\mathrm{s}^{35-37,40}$. 
Interestingly, zinc deficiency is reported to increase the risk of ventilator-induced injury in mice $^{41}$.

Moreover, zinc deficiency is associated with an increased risk of acute respiratory distress syndrome (ARDS) in human ${ }^{41}$.

These associations merit special attention concerning SARS-CoV-2 patients management ${ }^{15}$.

Therefore, we suggest that zinc deficiency should be investigated in a subgroup of SARS-CoV-2 patients at risk for developing hyperinflammatory complications ${ }^{15}$.

Additionally, we suggest that zinc correction might cooperate with $\mathrm{HCQ}$ in reducing the risk of SARS-CoV-2-related tissue-damaging cytokine storm, and we believe that such a probable synergy worth clinical exploration.

Zinc deficiency might deprive SARS-CoV-2 patients of additive Zinc-(Hydroxy)Chloroquine antiSARS effects

Several in-vitro studies had shown that zinc and zinc ionophores halt the RNA replication of different RNA viruses, including the ones involving human respiratory system ${ }^{42-49}$.

This large piece of the evidence inspired Velthuis et al. ${ }^{50}$ to test a similar possibility in two RNA viruses, including SARS-CoV.

The group discovered that both a candidate zinc ionophore (pyrithione) and Zinc itself could inhibit SARS-CoV replication as singe agents and better in combination. However and possibly due to low cellular uptake of Zinc, the optimal zinc concentration to efficiently inhibit the SARSCoV replication was relatively high ${ }^{50}$.

Notably, Velthuis et al..$^{50}$ could show that Zinc can directly disrupt the initiation of RNA synthesis by the SARS-CoV RNA-dependent RNA polymerase (RdRp) in-vitro, possibly via interacting with two zinc-binding pockets present in the SARS-CoV RdRp ${ }^{50}$.

It deserves to be mentioned that a Chinese group who has unraveled the SARS-CoV-2 RdRp genome, could not observe zinc ions being chelated at the two candidate pockets within the RdRp of SARS-CoV-2 ${ }^{51}$. The group addresses the discrepancy in their technical approach compared to the precedent works on SARS-CoV ${ }^{51}$.

Thanks to cancer research efforts, we learn a more exciting feature of CQ associated with Zinc. In 2014, a Chinese cancer study by Xue et al. reported that CQ increases zinc uptake in ovarian cancer cells and mediates zinc accumulation into the lysosomes of these cells ${ }^{52}$.

In contrast, a Korean study conducted by Seo et al. . ${ }^{53}$ partially contradicts findings of Xue et al., though in a different context and a separate cell line (adult retinal pigment epithelial cells). 
In contrast to the conclusion of Xue et al., Seo et al. ${ }^{53}$ reported that Chloroquine decreases the free zinc levels in lysosomes. However, they still observed some increased intracellular zinc levels upon $\mathrm{CQ}$ treatment compared to the control group.

Therefore, we acknowledge that whether $\mathrm{CQ} / \mathrm{HCQ}$ are global zinc ionophores mediating intracellular uptake of Zinc by cells of different origins, at this stage, should remain an open question and the subject of further investigation.

On the other hand, even if CQ or HCQ does not turn out to be zinc ionophore, it would still be possible that Zinc can exert an anti-SARS replication effect independent of CQ/HCQ. Patients with zinc deficiency would likely be deprived of this additive effect.

If further data suggests that $\mathrm{CQ} / \mathrm{HCQ}$ are zinc ionophores mediating zinc uptake into the SARSCoV-2 infected cells, one can postulate combining zinc supplements with $\mathrm{CQ} / \mathrm{HCQ}$ or at least zinc correction in zinc-deficient patients could be beneficial.

However, if the new data suggest that CQ/HCQ is interfering with zinc uptake into the SARSCoV-2 infected cells or in an organelle such as lysosomes- in line with findings of Seo et al. combining zinc correction or zinc supplementation with CQ/HCQ might be even highly essential.

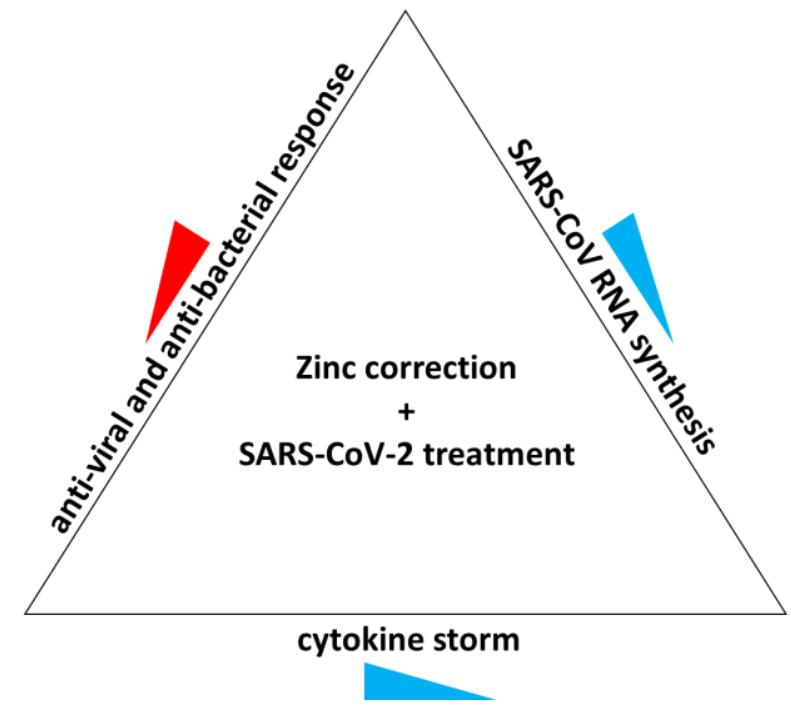

Figure 1 Three lines of evidence supporting the inclusion of zinc correction in different anti-SARSCoV-2 treatments including Hydroxychloroquine treatment

\section{Practicality}

There is no consensus and widely accepted guideline concerning zinc deficiency diagnosis and correction. Radioisotopes are a decent diagnostic tool, but they are costly. New biomarkers such as Linoleic Acid are emerging ${ }^{54-57}$. However, considering the vast population involved with SARS-CoV-2 and the high economic Burdon of such approaches, we propose a simple and cheap 
method to select zinc-deficient SARS-CoV-2 patients and include zinc correction in their treatment package.

\section{Zinc uptake and absorption:}

The Recommended Dietary Allowance (RDA) of zinc intake is $11 \mathrm{mg}$ and $8 \mathrm{mg} /$ day for adult males and females, respectively ${ }^{58}$.

According to an analysis of the National Health and Nutrition Examination Survey (NAHNES III), $35 \%-45 \%$ of adults above 60 years old have daily zinc intake below the recommended average 59.

The zinc transport protein family members that are apically positioned on the cell surface mediate zinc uptake.

Foods with a good source of Zinc include meat, fish, shellfish, legumes, nuts, seeds, eggs, and whole-grain cereals ${ }^{60}$.

Phytate, the hexaphosphate ester of inositol, due to its high polarity, strongly binds to divalent Zinc, thereby preventing its absorption ${ }^{61,62}$. Due to phytate-rich content, the zinc-bioavailability of vegan/vegetarian diets are less compared to omnivore diets ${ }^{62}$.

Nevertheless, unrefined phytate containing food such as whole-grain bread, despite higher phytate content, has higher zinc-bioavailability compared to refined ones like white bread, which have poor zinc content ${ }^{62}$.

\section{Evaluation of zinc status: Serum or plasma Zinc}

Plasma and serum zinc concentration are the most frequently used biomarkers to establish zinc status ${ }^{63}$.

Clinical effects of zinc deficiency can be existing despite normal zinc plasma concentration ${ }^{63}$. Conditions that lead to hypoalbuminemia also reduce plasma zinc concentrations, as Zinc is bound to the albumin in the plasma ${ }^{64}$. Therefore, it is essential to include albumin along with plasma zinc test and consider albumin-normalized zinc values to determine the zinc cut-off ${ }^{65}$.

Infections, fever, contraceptives, and pregnancy lower the plasma zinc while starvation and catabolism increase it ${ }^{63}$.

In particular and concerning SARS-CoV-2 patients, the status of two widely used biomarkers of inflammation ${ }^{31,66}$; c-reactive protein and procalcitonin, is linked to zinc status.

Interestingly, both these markers are inversely associated with serum zinc levels ${ }^{67,68}$, other evidence that zinc might have anti-hyper inflammatory effects in SARS-CoV-2 patients.

The interpretation of the concentration of plasma zinc must, therefore, take into account all the confounding factors ${ }^{69}$.

Plasma zinc concentrations dose-dependently respond to supplementation in patients with a low or moderate baseline, independent of gender and age ${ }^{69}$. 
On the other hand, Zinc deprivation results in a reduction in the plasma zinc concentrations.

The fact that plasma zinc concentrations are normally-distributed among healthy populations makes it useful as a zinc-deficiency marker ${ }^{63}$.

Frank T. et al. concluded that as plasma and urinary zinc concentrations are probably more precise indicators currently available, as opposed to indirect ones such as stunting, anemia, or iron deficiency. They suggest that direct indicators can be used to estimate the prevalence of zinc deficiency in populations ${ }^{57}$.

Cutoffs:

A revisit of the NHANES II data suggests cutoffs of serum zinc concentrations for assessing the fasting zinc status at 70 and $74 \mu \mathrm{g} / \mathrm{dL}$ for females and males over ten years old, respectively ${ }^{70}$.

\section{Treatment:}

Long term zinc supplementation should be very well-weighed ${ }^{71}$ as the therapeutic window of chronic zinc supplementation can relatively narrow.

In contrast, short term zinc supplementation may be less of a concern ${ }^{26}$.

The proposed dose of zinc (Znso4) injection for parenteral nutrition in metabolically stable adult patients is $3 \mathrm{mg} / \mathrm{day}^{72}$.

The maximum level of the excipient used in FDA-approved oral zinc tablets (excluding extended-release forms) is $25 \mathrm{mg}^{73}$.

The recommended oral daily dose for zinc correction in adults is $10-30 \mathrm{mg} / \mathrm{day}^{26,74}$.

Various forms of zinc supplements exist, including zinc gluconate, zinc citrate, zinc sulfate, and zinc acetate.

Rita Wegmüller et al. study demonstrated that zinc citrate could be as active as zinc gluconate in the prevention of zinc deficiency.

The high zinc content of zinc citrate, acceptable quality perception for the patients, the comparable bioavailability, and its low cost ${ }^{75}$ may make it a relevant form to be considered for zinc correction in zinc-deficient SARS-CoV-2 patients.

\section{Conclusion}

Taken all together, we believe there is a large piece of evidence that links antiviral response, zinc deficiency, and in particular, HC/HCQ therapy. At this stage, this evidence is far from being conclusive. However, such an indispensable and intriguing hint in the precedent literature 
justifies the clinical exploration of the possible link between zinc deficiency and response to $\mathrm{HC} / \mathrm{HCQ}$ and other treatments in SARS-CoV-2 patients.

\section{Author Contributions}

A.N and G.V developed the idea together and co-drafted the manuscript.

\section{Acknowledgments}

AN deeply appreciates Prof. Ilse Rooman's (LMMO-VUB) support to the present work.

\section{Grant Support}

A.N is the beneficiaries of a postdoctoral Fellowship from the Research Foundation-Flanders (FWO).

\section{Conflict of interest}

The authors declare that they have no conflicts of interest.

\section{References}

1. Cherry, J. D. \& Krogstad, P. SARS: the first pandemic of the 21st century. Pediatr. Res. 56, 1-5 (2004).

2. Andersen, K. G., Rambaut, A., Lipkin, W. I., Holmes, E. C. \& Garry, R. F. The proximal origin of SARS-CoV-2. Nat. Med. (2020) doi:10.1038/s41591-020-0820-9.

3. Zhang, T., Wu, Q. \& Zhang, Z. Probable Pangolin Origin of SARS-CoV-2 Associated with the COVID-19 Outbreak. Curr. Biol. (2020) doi:10.1016/j.cub.2020.03.022.

4. Lam, T. T.-Y. et al. Identifying SARS-CoV-2 related coronaviruses in Malayan pangolins. Nature (2020) doi:10.1038/s41586-020-2169-0.

5. Savarino, A., Boelaert, J. R., Cassone, A., Majori, G. \& Cauda, R. Effects of chloroquine on viral infections: an old drug against today's diseases? Lancet. Infect. Dis. 3, 722-727 (2003).

6. Keyaerts, E., Vijgen, L., Maes, P., Neyts, J. \& Van Ranst, M. In vitro inhibition of severe acute respiratory syndrome coronavirus by chloroquine. Biochem. Biophys. Res.

Commun. 323, 264-268 (2004).

7. Vincent, M. J. et al. Chloroquine is a potent inhibitor of SARS coronavirus infection and spread. Virol. J. 2, 69 (2005).

8. Wang, M. et al. Remdesivir and chloroquine effectively inhibit the recently emerged 
novel coronavirus (2019-nCoV) in vitro. Cell Res. 30, 269-271 (2020).

9. Liu, J. et al. Hydroxychloroquine, a less toxic derivative of chloroquine, is effective in inhibiting SARS-CoV-2 infection in vitro. Cell Discov. 6, 16 (2020).

10. Yao, X. et al. In Vitro Antiviral Activity and Projection of Optimized Dosing Design of Hydroxychloroquine for the Treatment of Severe Acute Respiratory Syndrome Coronavirus 2 (SARS-CoV-2). Clin. Infect. Dis. (2020) doi:10.1093/cid/ciaa237.

11. Schrezenmeier, E. \& Dorner, T. Mechanisms of action of hydroxychloroquine and chloroquine: implications for rheumatology. Nat. Rev. Rheumatol. 16, 155-166 (2020).

12. Baughman, R. P., Lower, E. E. \& du Bois, R. M. Sarcoidosis. Lancet (London, England) 361, 1111-1118 (2003).

13. Sharma, O. P. Effectiveness of Chloroquine and Hydroxychloroquine in Treating Selected Patients With Sarcoidosis With Neurological Involvement. Arch. Neurol. 55, 1248-1254 (1998).

14. Zhou, D., Dai, S.-M. \& Tong, Q. COVID-19: a recommendation to examine the effect of hydroxychloroquine in preventing infection and progression. J. Antimicrob. Chemother. (2020) doi:10.1093/jac/dkaa114.

15. Mehta, P. et al. COVID-19: consider cytokine storm syndromes and immunosuppression. Lancet (London, England) (2020) doi:10.1016/S0140-6736(20)30628-0.

16. Wu, S.-F. et al. Hydroxychloroquine inhibits CD154 expression in CD4(+) T lymphocytes of systemic lupus erythematosus through NFAT, but not STAT5, signaling. Arthritis Res. Ther. 19, 183 (2017).

17. Dewitte, A. et al. CD154 Induces Interleukin-6 Secretion by Kidney Tubular Epithelial Cells under Hypoxic Conditions: Inhibition by Chloroquine. Mediators Inflamm. 2020, 6357046 (2020).

18. Gao, J., Tian, Z. \& Yang, X. Breakthrough: Chloroquine phosphate has shown apparent efficacy in treatment of COVID-19 associated pneumonia in clinical studies. Biosci. Trends 14, 72-73 (2020).

19. Cortegiani, A., Ingoglia, G., Ippolito, M., Giarratano, A. \& Einav, S. A systematic review on the efficacy and safety of chloroquine for the treatment of COVID-19. J. Crit. Care (2020) doi:10.1016/j.jcrc.2020.03.005.

20. Gautret, P. et al. Hydroxychloroquine and azithromycin as a treatment of COVID-19: results of an open-label non-randomized clinical trial. Int. J. Antimicrob. Agents 105949 (2020) doi:10.1016/j.ijantimicag.2020.105949.

21. (Nurarif \& Kusuma, 2016). 済無No Title No Title. J. Chem. Inf. Model. 53, 1689-1699 (2013).

22. Al., P. G. and. Clinical and microbiological effect of a combination of hydroxychloroquine 
and azithromycin in 80 COVID-19 patients with at least a six-day follow up: an observational study. $\mathbf{8}$.

23. Chen, Z. et al. Efficacy of hydroxychloroquine in patients with COVID-19: results of a randomized clinical trial. medRxiv 2020.03.22.20040758 (2020) doi:10.1101/2020.03.22.20040758.

24. PRASAD, A. S., HALSTED, J. A. \& NADIMI, M. Syndrome of iron deficiency anemia, hepatosplenomegaly, hypogonadism, dwarfism and geophagia. Am. J. Med. 31, 532-546 (1961).

25. Prasad, A. S. et al. Zinc supplementation decreases incidence of infections in the elderly: effect of zinc on generation of cytokines and oxidative stress. Am. J. Clin. Nutr. 85, 837844 (2007).

26. Roohani, N., Hurrell, R., Kelishadi, R. \& Schulin, R. Zinc and its importance for human health: An integrative review. J. Res. Med. Sci. 18, 144-157 (2013).

27. Read, S. A., Obeid, S., Ahlenstiel, C. \& Ahlenstiel, G. The Role of Zinc in Antiviral Immunity. Adv. Nutr. 10, 696-710 (2019).

28. Chen, Y. \& Li, L. SARS-CoV-2: virus dynamics and host response. Lancet. Infect. Dis. (2020) doi:10.1016/S1473-3099(20)30235-8.

29. Bhutta, Z. A. et al. Prevention of diarrhea and pneumonia by zinc supplementation in children in developing countries: pooled analysis of randomized controlled trials. Zinc Investigators' Collaborative Group. J. Pediatr. 135, 689-697 (1999).

30. Prasad, A. S. Zinc in human health: effect of zinc on immune cells. Mol. Med. 14, 353-357 (2008).

31. Zhang, J. et al. Therapeutic and triage strategies for 2019 novel coronavirus disease in fever clinics. The Lancet. Respiratory medicine vol. 8 e11-e12 (2020).

32. Sanna, A., Firinu, D., Zavattari, P. \& Valera, P. Zinc Status and Autoimmunity: A Systematic Review and Meta-Analysis. Nutrients 10, (2018).

33. von Bulow, V. et al. Zinc-dependent suppression of TNF-alpha production is mediated by protein kinase A-induced inhibition of Raf-1, I kappa B kinase beta, and NF-kappa B. J. Immunol. 179, 4180-4186 (2007).

34. Suzuki, T., Katsumata, S.-I., Matsuzaki, H. \& Suzuki, K. Dietary zinc supplementation increased TNF $\alpha$ and IL1 $\beta$-induced RANKL expression, resulting in a decrease in bone mineral density in rats. J. Clin. Biochem. Nutr. 58, 48-55 (2016).

35. Gruber, K. et al. Zinc deficiency adversely influences interleukin-4 and interleukin-6 signaling. J. Biol. Regul. Homeost. Agents 27, 661-671 (2013).

36. Wong, C. P., Rinaldi, N. A. \& Ho, E. Zinc deficiency enhanced inflammatory response by increasing immune cell activation and inducing IL6 promoter demethylation. Mol. Nutr. 
Food Res. 59, 991-999 (2015).

37. Prasad, A. S. Effects of zinc deficiency on Th1 and Th2 cytokine shifts. J. Infect. Dis. 182 Suppl, S62-8 (2000).

38. Cipriano, C. et al. Polymorphisms in MT1a gene coding region are associated with longevity in Italian Central female population. Biogerontology 7, 357-365 (2006).

39. Mariani, E. et al. Effect of zinc supplementation on plasma IL-6 and MCP-1 production and NK cell function in healthy elderly: interactive influence of +647 MT1a and -174 IL-6 polymorphic alleles. Exp. Gerontol. 43, 462-471 (2008).

40. Kitabayashi, C. et al. Zinc suppresses Th17 development via inhibition of STAT3 activation. Int. Immunol. 22, 375-386 (2010).

41. Boudreault, F. et al. Zinc deficiency primes the lung for ventilator-induced injury. $\mathrm{JCI}$ insight 2, (2017).

42. Krenn, B. M. et al. Antiviral activity of the zinc ionophores pyrithione and hinokitiol against picornavirus infections. J. Virol. 83, 58-64 (2009).

43. Lanke, K., Krenn, B. M., Melchers, W. J. G., Seipelt, J. \& van Kuppeveld, F. J. M. PDTC inhibits picornavirus polyprotein processing and RNA replication by transporting zinc ions into cells. J. Gen. Virol. 88, 1206-1217 (2007).

44. Polatnick, J. \& Bachrach, H. L. Effect of zinc and other chemical agents on foot-andmouth-disease virus replication. Antimicrob. Agents Chemother. 13, 731-734 (1978).

45. Korant, B. D., Kauer, J. C. \& Butterworth, B. E. Zinc ions inhibit replication of rhinoviruses. Nature 248, 588-590 (1974).

46. $\mathrm{Si}$, X. et al. Pyrrolidine dithiocarbamate reduces coxsackievirus B3 replication through inhibition of the ubiquitin-proteasome pathway. J. Virol. 79, 8014-8023 (2005).

47. Gaudernak, E., Seipelt, J., Triendl, A., Grassauer, A. \& Kuechler, E. Antiviral effects of pyrrolidine dithiocarbamate on human rhinoviruses. J. Virol. 76, 6004-6015 (2002).

48. Suara, R. O. \& Crowe, J. E. J. Effect of zinc salts on respiratory syncytial virus replication. Antimicrob. Agents Chemother. 48, 783-790 (2004).

49. Uchide, N., Ohyama, K., Bessho, T., Yuan, B. \& Yamakawa, T. Effect of antioxidants on apoptosis induced by influenza virus infection: inhibition of viral gene replication and transcription with pyrrolidine dithiocarbamate. Antiviral Res. 56, 207-217 (2002).

50. te Velthuis, A. J. W. et al. Zn(2+) inhibits coronavirus and arterivirus RNA polymerase activity in vitro and zinc ionophores block the replication of these viruses in cell culture. PLoS Pathog. 6, e1001176 (2010).

51. Gao, Y. et al. Structure of RNA-dependent RNA polymerase from 2019-nCoV, a major antiviral drug target. bioRxiv 2020.03.16.993386 (2020) doi:10.1101/2020.03.16.993386. 
52. Xue, J. et al. Chloroquine is a zinc ionophore. PLoS One 9, e109180 (2014).

53. Seo, B.-R., Lee, S.-J., Cho, K. S., Yoon, Y. H. \& Koh, J.-Y. The zinc ionophore clioquinol reverses autophagy arrest in chloroquine-treated ARPE-19 cells and in APP/mutant presenilin-1-transfected Chinese hamster ovary cells. Neurobiol. Aging 36, 3228-3238 (2015).

54. King, J. C. et al. Biomarkers of Nutrition for Development (BOND)-Zinc Review. J. Nutr. 146, 858S-885S (2015).

55. Knez, M., Stangoulis, J. C. R., Glibetic, M. \& Tako, E. The Linoleic Acid: Dihomo- $\gamma$-Linolenic Acid Ratio (LA:DGLA)-An Emerging Biomarker of Zn Status. Nutrients 9, (2017).

56. Tran, C. D., Gopalsamy, G. L., Mortimer, E. K. \& Young, G. P. The potential for zinc stable isotope techniques and modelling to determine optimal zinc supplementation. Nutrients 7, 4271-4295 (2015).

57. Wieringa, F. T., Dijkhuizen, M. A., Fiorentino, M., Laillou, A. \& Berger, J. Determination of zinc status in humans: which indicator should we use? Nutrients 7, 3252-3263 (2015).

58. Intakes, D. R. et al. Dietary reference intakes for vitamin A, vitamin $K$, arsenic, boron, chromium, copper, iodine, iron, manganese, molybdenum, nickel, silicon, vanadium, and zinc : a report of the Panel on Micronutrients ... [et al.], Standing Committee on the Scientific Eva. (2002).

59. Briefel, R. R. et al. Zinc intake of the U.S. population: findings from the third National Health and Nutrition Examination Survey, 1988-1994. J. Nutr. 130, 1367S-73S (2000).

60. Cousins, R. J. Gastrointestinal factors influencing zinc absorption and homeostasis. Int. J. Vitam. Nutr. Res. Int. Zeitschrift fur Vitamin- und Ernahrungsforschung. J. Int. Vitaminol. Nutr. 80, 243-248 (2010).

61. Mertz, W. Protein Nutrition and Mineral Absorption. The American Journal of Clinical Nutrition vol. 54 (CRC press, 1991).

62. Hunt, J. R. Bioavailability of iron, zinc, and other trace minerals from vegetarian diets. Am. J. Clin. Nutr. 78, 633S-639S (2003).

63. Sandstr, B. Diagnosis of zinc de fi ciency and excess in individuals. 22, 133-137 (2001).

64. Blindauer, C. A. et al. Structure, properties, and engineering of the major zinc binding site on human albumin. J. Biol. Chem. 284, 23116-23124 (2009).

65. King, J. C. Yet Again, Serum Zinc Concentrations Are Unrelated to Zinc Intakes. J. Nutr. 148, 1399-1401 (2018).

66. Lippi, G. \& Plebani, M. Procalcitonin in patients with severe coronavirus disease 2019 (COVID-19): A meta-analysis. Clinica chimica acta; international journal of clinical chemistry vol. 505 190-191 (2020).

67. Bao, B. et al. Zinc decreases C-reactive protein, lipid peroxidation, and inflammatory 
cytokines in elderly subjects: a potential implication of zinc as an atheroprotective agent. Am. J. Clin. Nutr. 91, 1634-1641 (2010).

68. Hoeger-Schäfer, J. et al. Persistent low serum zinc is associated with recurrent sepsis in critically ill patients - A pilot study. PLoS One 12, (2017).

69. Lowe, N. M., Fekete, K. \& Decsi, T. Methods of assessment of zinc status in humans: a systematic review. Am. J. Clin. Nutr. 89, 2040S-2051S (2009).

70. Hotz, C., Peerson, J. M. \& Brown, K. H. Suggested lower cutoffs of serum zinc concentrations for assessing zinc status: reanalysis of the second National Health and Nutrition Examination Survey data (1976-1980). Am. J. Clin. Nutr. 78, 756-764 (2003).

71. Maret, W. \& Sandstead, H. H. Zinc requirements and the risks and benefits of zinc supplementation. J. Trace Elem. Med. Biol. 20, 3-18 (2006).

72. Review, M. CENTER FOR DRUG EVALUATION AND MULTI-DISCIPLINE REVIEW Summary Review Office Director Clinical Statistical Clinical Pharmacology. Acalabrutinib NDA (2016).

73. Research, F. for D. E. and et al. No Title. https://www.accessdata.fda.gov/scripts/cder/iig/index.cfm?event=browseByLetter.page \&Letter=Z (2020).

74. Gupta, M., Mahajan, V. K., Mehta, K. S. \& Chauhan, P. S. Zinc therapy in dermatology: a review. Dermatol. Res. Pract. 2014, 709152 (2014).

75. Wegmuller, R., Tay, F., Zeder, C., Brnic, M. \& Hurrell, R. F. Zinc absorption by young adults from supplemental zinc citrate is comparable with that from zinc gluconate and higher than from zinc oxide. J. Nutr. 144, 132-136 (2014). 